\title{
MRI-Based Methods for Spinal Cord Atrophy Evaluation: A Comparison of Cervical Cord Cross-Sectional Area, Cervical Cord Volume, and Full Spinal Cord Volume in Patients with Aquaporin-4 Antibody Seropositive Neuromyelitis Optica Spectrum Disorders
}

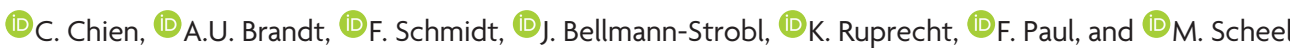

\begin{abstract}
BACKGROUND AND PURPOSE: Measures for spinal cord atrophy have become increasingly important as imaging biomarkers in the assessment of neuroinflammatory diseases, especially in neuromyelitis optica spectrum disorders. The most commonly used method, mean upper cervical cord area, is relatively easy to measure and can be performed on brain MRIs that capture cervical myelon. Measures of spinal cord volume (eg, cervical cord volume or total cord volume) require longer scanning and more complex analysis but are potentially better suited as spinal cord atrophy measures. This study investigated spinal cord atrophy measures in a cohort of healthy subjects and patients with aquaporin-4 antibody seropositive neuromyelitis optica spectrum disorders and evaluated the discriminatory performance of mean upper cervical cord cross-sectional area compared with cervical cord volume and total cord volume.
\end{abstract}

MATERIALS AND METHODS: Mean upper cervical cord area, cervical cord volume, and total cord volume were measured using 3T MRIs from healthy subjects $(n=19)$ and patients with neuromyelitis optica spectrum disorders $(n=30)$. Group comparison and receiver operating characteristic analyses between healthy controls and patients with neuromyelitis optica spectrum disorders were performed.

RESULTS: Mean upper cervical cord area, cervical cord volume, and total cord volume measures showed similar and highly significant group differences between healthy control subjects and patients with neuromyelitis optica spectrum disorders $(P<.01$ for all). All 3 measures showed similar receiver operating characteristic-area under the curve values (mean upper cervical cord area $=0.70$, cervical cord volume $=0.75$, total cord volume $=0.77$ ) with no significant difference between them. No associations among mean upper cervical cord cross-sectional area, cervical cord volume, or total cord volume with disability measures were found.

CONCLUSIONS: All 3 measures showed similar discriminatory power between healthy control and neuromyelitis optica spectrum disorders groups. Mean upper cervical cord area is easier to obtain compared with cervical cord volume and total cord volume and can be regarded as an efficient representative measure of spinal cord atrophy in the neuromyelitis optica spectrum disorders context.

ABBREVIATIONS: AQP4-Ab+ = aquaporin-4 antibody seropositive; $\mathrm{CCV}=$ cervical cord volume; $\mathrm{EDSS}=$ Expanded Disability Status Scale; MUCCA = mean upper cervical cord area; NMOSD = neuromyelitis optica spectrum disorders; $\mathrm{SCA}=$ spinal cord atrophy; $\mathrm{TCV}=$ total cord volume

$\mathbf{S}_{\text {ind }}^{\text {end }}$ everal imaging biomarkers have been established in treatment trials for neuroinflammatory diseases. The most prominent example is T2 lesion count and/or volume in multiple sclerosis. ${ }^{1}$ In neuromyelitis optica spectrum disorders (NMOSD), no such imaging biomarkers have been established yet. In most patients with NMOSD, standard brain MR imaging shows only nonspe-

Received December 3, 2017; accepted after revision March 13, 2018.

From the NeuroCure Clinical Research Center (C.C., A.U.B., F.S., J.B.-S., F.P. M.S.) and Departments of Neurology (F.S., K.R., F.P.) and Neuroradiology (M.S.), Charité-Universitätsmedizin Berlin, Berlin, Germany; and Experimental and Clinical Research Center (J.B.-S., F.P.), Max Delbrück Center for Molecular Medicine and CharitéUniversitätsmedizin Berlin, Berlin, Germany.

F. Paul and M. Scheel were equally contributing senior authors.

The data collection and analyses for this study were funded by the Deutsche Forschungsgemeinschaft, German Research Foundation (grant No. Exc 257). cific lesions. ${ }^{2,3}$ However NMOSD has a predilection for affecting the optic nerves and spinal cord. ${ }^{4-6}$

Spinal cord atrophy (SCA) can appear after longitudinally extensive transverse myelitis ${ }^{7-9}$ and has therefore been suggested as an imaging biomarker for disease severity and treatment response in NMOSD. Previous studies have demonstrated that SCA can be associated with longer disease duration and more severe disability and can potentially indicate poor prognosis in NMOSD. ${ }^{10,11}$

The SCA measurement most commonly used is the mean upper cervical cord area (MUCCA). It is measured as the cross-

\footnotetext{
Please address correspondence to Friedemann Paul, MD, NeuroCure Clinical Research Center, Charité-Universitätsmedizin Berlin, Charitéplatz 1, 10117 Berlin, Germany; e-mail: friedemann.paul@charite.de

- Indicates open access to non-subscribers at www.ajnr.org

http://dx.doi.org/10.3174/ajnr.A5665
} 
sectional area of the spinal cord at the level between the $\mathrm{C} 2$ and C3 vertebrae. ${ }^{12-15}$ Although longitudinally extensive transverse myelitis predominantly affects the cervical and upper thoracic spinal cord, the lower thoracic and lumbar cord in patients with NMOSD is often affected as well. ${ }^{16,17}$ Hence, the main disadvantage of MUCCA is that though it can be easily obtained and measured, it covers only a very small fraction of the spinal cord. Therefore, quantification of the total cord volume (TCV) is theoretically beneficial for a more precise assessment of SCA.

The main disadvantage of assessing TCV is that it requires extra scan time and a more complex and time-consuming analysis procedure. As a compromise between MUCCA and TCV quantification, cervical cord volume (CCV) could be measured.

In theory, TCV should best discriminate between patients with NMOSD and healthy control subjects and should also show a stronger association with clinical measures of disability. Our hypothesis was that TCV best reflects full SCA. Our study compares the discriminatory power of MUCCA, CCV, and TCV between patients with NMOSD and a healthy control group and investigates associations of MUCCA, CCV, and TCV with clinical disability measures.

\section{MATERIALS AND METHODS Patients and Subjects}

Clinical, demographic, and MR imaging data from 30 patients with NMOSD and 19 healthy control subjects were included (Table 1) in this study. These data were acquired from an ongoing longitudinal prospective observational cohort study, which was approved by the local ethics committee.

All patients and subjects provided written informed consent. The study was conducted in accordance with the Declaration of Helsinki in its current applicable version and applicable countryspecific laws.
All patients had a definite diagnosis of NMOSD according to current panel criteria ${ }^{7}$ and were aquaporin- 4 antibody seropositive $(\mathrm{AQP} 4-\mathrm{Ab}+) .{ }^{18} \mathrm{We}$ limited the patient cohort to those with AQP4-Ab + NMOSD only, to ensure a homogeneous patient cohort with a comparable mechanism of spinal cord damage. All patients with AQP4-Ab+ NMOSD except for 2 (93\%) had an attack history of myelitis. Clinical assessments consisted of the Expanded Disability Status Scale (EDSS), averaged timed 25-foot walk test time, and the averaged 9-hole peg test time.

\section{MR Imaging Acquisition}

All MR imaging scans were performed on a 3T (Magnetom Trio Tim; Siemens, Erlangen, Germany) scanner. The MR imaging protocol for this study included the following: 1) a T1-weighted 3D magnetization prepared rapid acquisition of gradient echo brain MR imaging $\left(1 \times 1 \times 1 \mathrm{~mm}^{3}\right.$ resolution, $\mathrm{TR}=1900 \mathrm{~ms}, \mathrm{TE}=3.03 \mathrm{~ms}$, in-plane resolution $=1 \times 1 \mathrm{~mm}$ ) including the upper cervical cord, and 2) sagittally oriented $2 \mathrm{D}$ T2-weighted sequences (slice thickness $=2$ $\mathrm{mm}$, gap size $=0.2 \mathrm{~mm}, \mathrm{TR}=3500 \mathrm{~ms}, \mathrm{TE}=101 \mathrm{~ms}$, in-plane resolution $=1.2 \times 0.9 \mathrm{~mm}$ ) at the cervical, thoracic, and lumbar levels.

\section{Spinal Cord Analysis}

All spinal cord measurements were performed with Jim software (Version 7.0; http://www.xinapse.com/home.php). Jim software applies a semiautomatic active surface model, which is based on spinal cord surface parametrization, yielding reproducible measurements of cord cross-sectional areas. This method is also applicable in full spinal cord segmentation of 2D multislice T2-weighted MRI, giving volume measurements of specified spinal cord regions such as the cervical cord or full spinal cord. ${ }^{19}$ Because this method has been validated for area and volumetric measurements in both research and clinical settings, we chose the Jim software for this study. ${ }^{20-22}$
Table 1: Demographics and clinical characteristics

\begin{tabular}{lccc}
\hline & HCs & Patients with NMOSD & $P$ Value \\
\hline No. & 19 & 30 & - \\
Sex (F/M) (\% female) & $16: 3(84 \%)$ & $27: 3(90 \%)$ & $.56^{\mathrm{a}}$ \\
Mean age (range) (yr) & $41.6(24-68)$ & $46.5(18-70)$ & $.23^{\mathrm{b}}$ \\
Median EDSS score (range) & - & $3.8(0-6.5)$ & - \\
Mean disease duration (range) (yr) & - & $5.7(0.5-18.5)$ & - \\
\hline
\end{tabular}

Note:-HCs indicates healthy controls.

${ }^{\mathrm{a}} \chi^{2}$.

${ }^{\mathrm{b}}$ Two-sample $t$ test.

\section{Mean Upper Cervical Cord Area}

MUCCA was measured in 3D MPRAGE images by averaging the cross-sectional areas from 5 consecutive slices. Following standard convention, the C2-C3 intervertebral space was used as a horizontal reference level for the middle slice. ${ }^{12}$

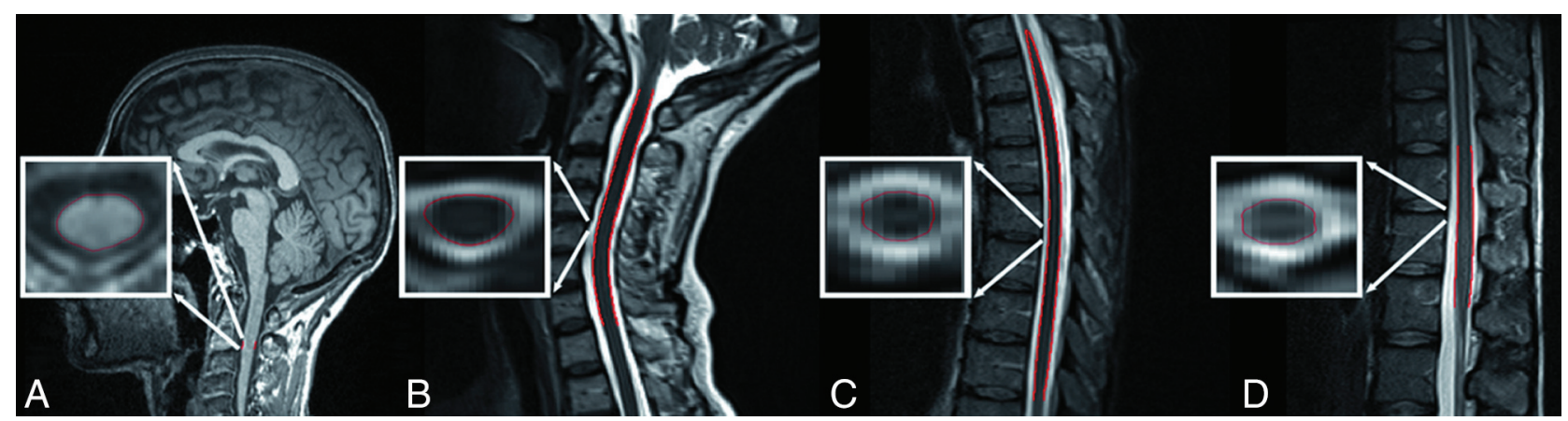

FIG 1. Sample segmentation of MUCCA $(A), C C V(B)$, the thoracic spinal cord $(C)$, and the lumbar spinal cord $(D)$. TCV is calculated from the sum of $B, C$, and $D$. 


\section{Cervical Cord Volume}

CCV was measured in a cervical spinal cord 2D T2-weighted sagittal MR imaging sequence. Images were reoriented to the axial orientation and interpolated with multiplanar reconstruction. The centerline of the cord was manually marked at 4 levels along the spinal cord from the tip of the dens to the rostral border of the T1 vertebral body. Subsequent automatic volumetric analysis of the cervical spinal cord was measured with the active surface model as implemented in Jim 7.0 software.

\section{Total Cord Volume}

TCV was measured using spinal cord sagittal 2D T2-weighted MR imaging sequences at 3 different levels: cervical, thoracic, and lumbar. All preprocessing by reorientation, multiplanar reconstruction, and centerline indication was the same as with the CCV. Therefore, the volumetric analysis of the full spinal cord required segmentation into 3 parts: 1 ) the cervical cord from the tip of the dens to the rostral border of the T1 vertebral body, 2) the thoracic cord from the rostral border of the T1 vertebral body to the T11 vertebral body rostral border, and 3) the lumbar cord from the T11 vertebral body rostral border to the conus tip. The TCV was calculated by adding all segmented volumes together.

Figure 1 shows a sample segmentation performed with the active surface model for MUCCA, CCV, and the thoracic and lumbar cords.

\section{Statistical Analysis}

Proportional group differences in sex were tested with a $\chi^{2}$ test. For group comparison of continuous measurements (age, MUCCA, CCV, TCV), a Welch 2-sampled $t$ test was used.

Association analysis of MUCCA, CCV, and TCV with each other and with clinical performance measures (averaged timed 25-foot walk and dominant and nondominant hand 9-hole peg test times) was performed with the Pearson correlation test and for EDSS with the Spearman rank correlation test. Further group discriminatory performance was evaluated with receiver operating characteristic analysis including area under the curve comparison using the DeLong method. ${ }^{23}$

All statistical analyses and plots were produced using $\mathrm{R}$ software (http://www.r-project.org/) ${ }^{24}$ with the tidyverse, ${ }^{25}$ ggpubr, ${ }^{26}$ and pROC packages. ${ }^{27}$

\section{RESULTS}

\section{Demographics}

Table 1 shows the demographic data of the study cohort. No significant differences were found between groups in sex or age.

\section{Group Comparison and Receiver Operating Characteristic Analysis}

Table 2 and Figs 2 and 3 summarize the group comparison analysis and spinal cord association analyses between patients with NMOSD and healthy controls for MUCCA, CCV, and TCV. All 3 measures show a clear and significant difference between each group and significant associations with each other.

Differentiation between healthy control and NMOSD group spinal cord measures are evident, as shown in Fig 2. MUCCA, $\mathrm{CCV}$, and TCV have comparable area under the curve values in the receiver operating characteristic analysis, as shown in Fig 4. Area under the curve values were tested for a statistically significance difference using the DeLong method, ${ }^{23}$ yielding no increased performance of any spinal cord measure over the other.

Table 2: Spinal cord measurements

\begin{tabular}{lccc}
$\begin{array}{c}\text { Spinal } \\
\text { Cord Measure }\end{array}$ & $\begin{array}{c}\text { HCs } \\
\text { (Mean } \pm \text { SD) }\end{array}$ & $\begin{array}{c}\text { Patients with NMOSD } \\
\text { (Mean } \pm \text { SD) }\end{array}$ & $\begin{array}{c}\text { T Statistic; } \\
\text { P Value }\end{array}$ \\
\hline MUCCA $\left(\mathrm{mm}^{2}\right)$ & $73.3 \pm 5.51$ & $68.5 \pm 7.06$ & $2.70 ; .009^{\mathrm{a}}$ \\
CCV $(\mathrm{mL})$ & $7.52 \pm 0.92$ & $6.61 \pm 0.96$ & $3.33 ; .002^{\mathrm{a}}$ \\
TCV $(\mathrm{mL})$ & $20.1 \pm 2.37$ & $17.6 \pm 2.21$ & $3.69 ;<.001^{\mathrm{a}}$ \\
\hline
\end{tabular}

Note:- HCs indicates healthy controls.

a Statistical difference from HCs $(P<.01)$.

MUCCA

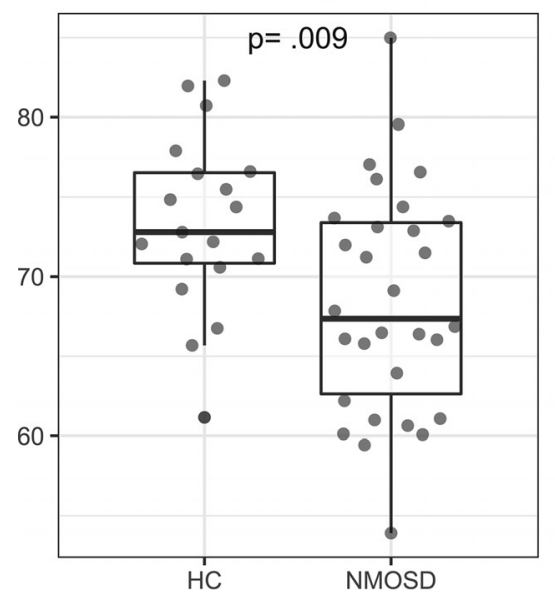

CCV

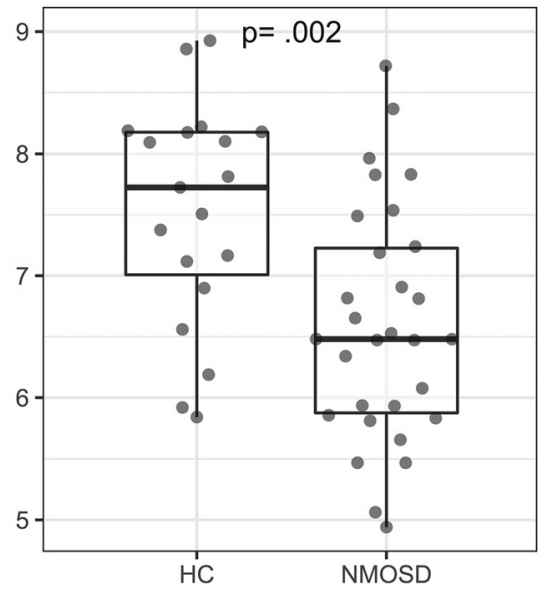

Associations with Clinical Disability Associations of MUCCA, CCV, and TCV with the EDSS and the pyramidal functional system score of the EDSS ${ }^{28}$ all showed nonsignificant associations (Fig 5 and Table 3). No spinal cord measures showed significant associa-

TCV

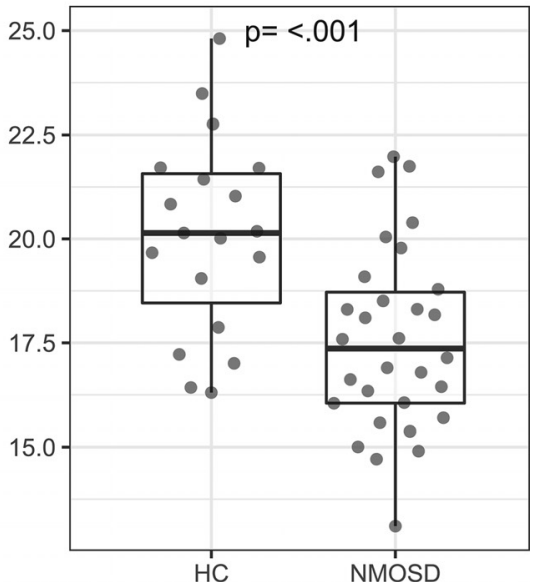

FIG 2. Group differences between healthy control $(\mathrm{HC})$ subjects and patients with NMOSD for MUCCA in square millimeters, CCV and TCV, in milliliters. 

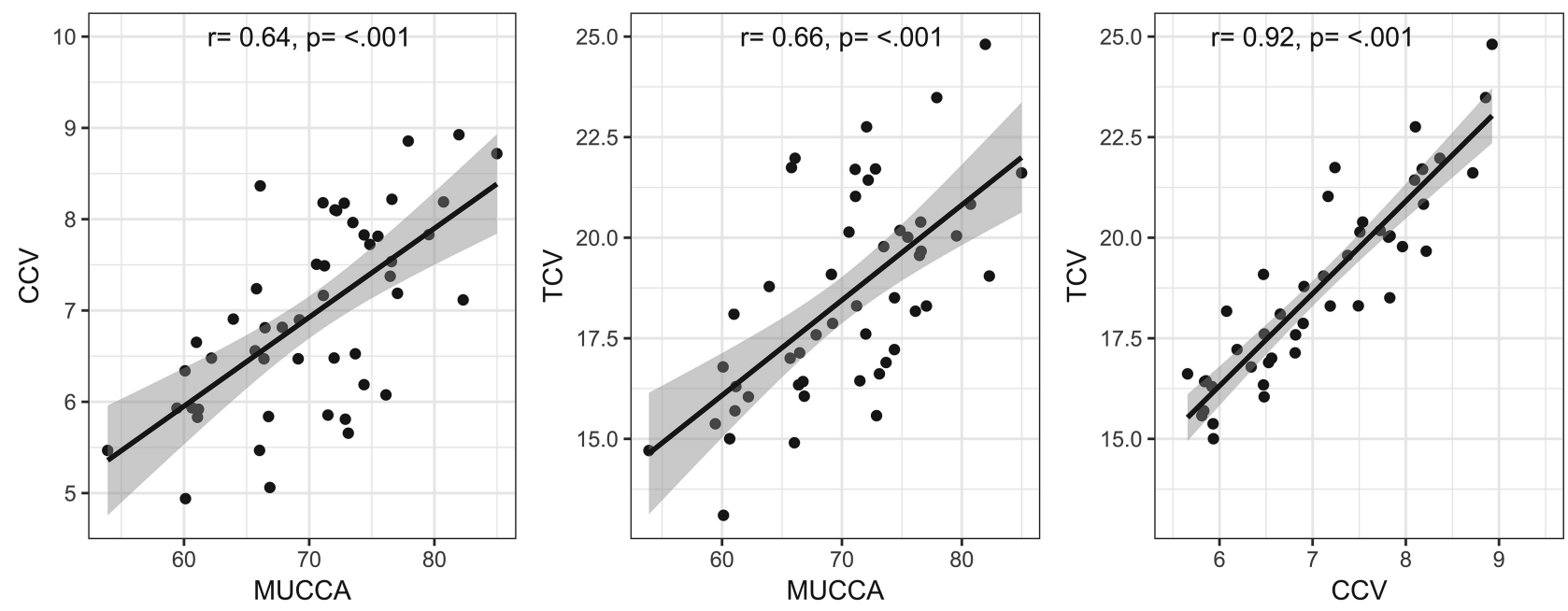

FIG 3. Pearson correlation tests of each spinal cord measure for healthy controls and patients with NMOSD combined. MUCCA is shown in square millimeters; CCV and TCV, in milliliters.
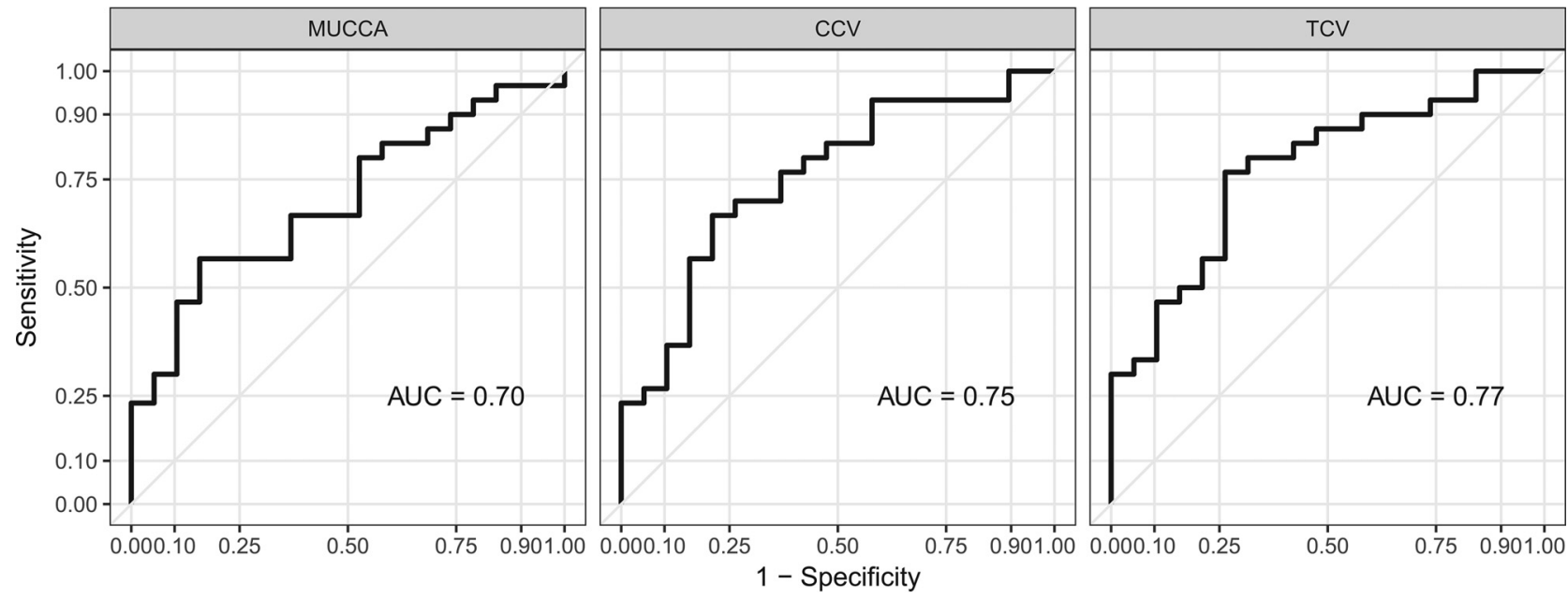

FIG 4. Receiver operating characteristic analysis for MUCCA, CCV, and TCV indicates good sensitivity (true-positive fraction) and specificity (false-positive fraction) for spinal cord atrophy measures in NMOSD. AUC indicates area under the curve.
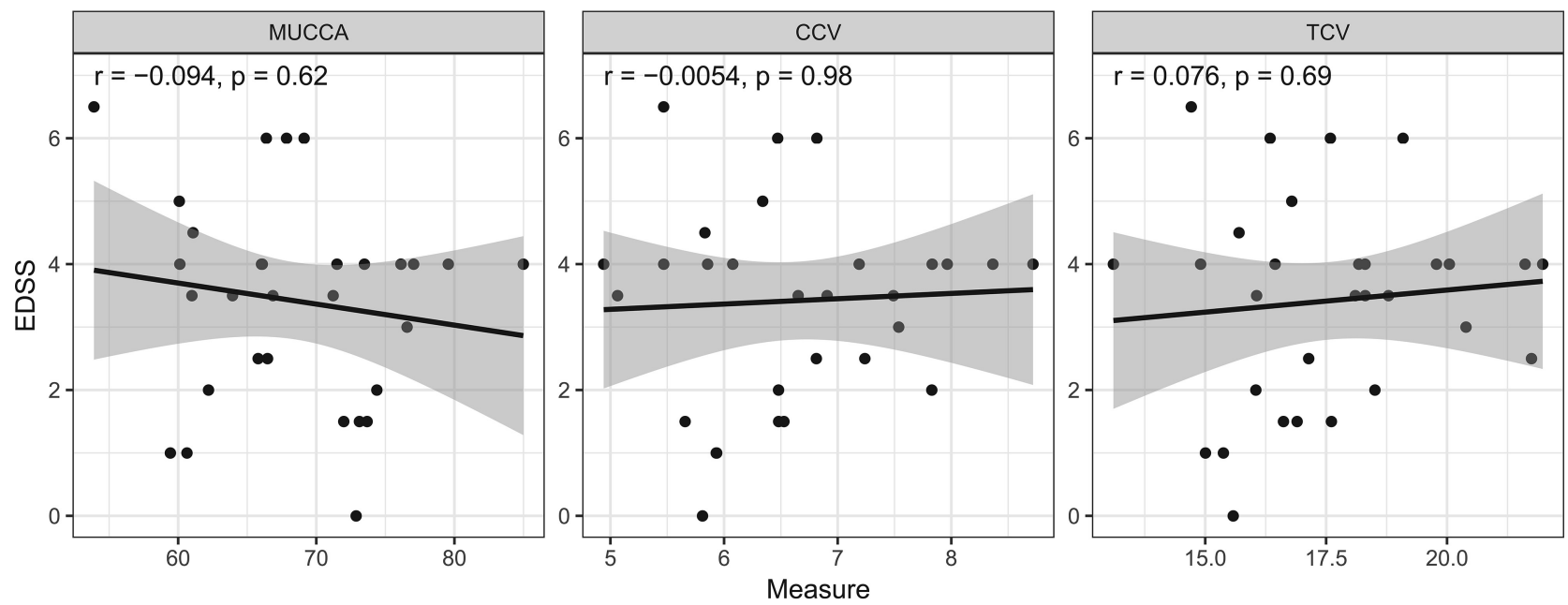

FIG 5. Associations of each spinal cord measure with the Expanded Disability Status Scale score. The x-axis shows measurements for MUCCA in square millimeters, CCV and TCV, in milliliters. 
Table 3: Associations of spinal cord measurements with clinical disability

\begin{tabular}{|c|c|c|c|c|}
\hline \multirow{2}{*}{$\begin{array}{c}\text { Spinal } \\
\text { Cord Measure }\end{array}$} & \multirow{2}{*}{$\begin{array}{l}\text { Pyramidal Functional } \\
\text { System Score }\end{array}$} & \multirow{2}{*}{$\begin{array}{l}\text { Average Timed } \\
25-\text { Foot Walk }^{\mathrm{b}}\end{array}$} & \multicolumn{2}{|c|}{ 9-Hole Peg Test ${ }^{b}$} \\
\hline & & & Average Dominant Hand & Average Nondominant Hand \\
\hline MUCCA & $R=-0.31, P=.1$ & $R=-0.22, P=.25$ & $R=-0.009, P=.97$ & $R=-0.12, P=.56$ \\
\hline $\mathrm{CCV}$ & $R=-0.058, P=.76$ & $R=-0.20, P=.30$ & $R=-0.085, P=.67$ & $R=0.005, P=.98$ \\
\hline $\mathrm{TCV}$ & $R=0.016, P=.93$ & $R=-0.13, P=.50$ & $R=0.046, P=.82$ & $R=0.13, P=.50$ \\
\hline
\end{tabular}

${ }^{a}$ Nonparametric Spearman rank correlation coefficient

b Pearson correlation test.

tions with the averaged timed 25-foot walk or 9-hole peg tests (Table 3).

\section{DISCUSSION}

This study investigated 3 different MR imaging-based SCA measures for discriminatory performance in a cohort of patients with NMOSD and healthy control subjects. We demonstrated that all 3 types of quantification could significantly differentiate these 2 groups. In a receiver operating characteristic analysis, all measures showed similar area under the curve values in the range of $0.70-0.77$, in which TCV had the highest value on a descriptive level. In a statistical comparison, these values were not significantly different. MUCCA, CCV, and TCV did not correlate with clinical disability, as measured by the EDSS, the pyramidal functional system score, and the averaged timed 25 -foot walk and 9-hole peg tests.

The observed significant difference in MUCCA between groups is in line with results from other studies. ${ }^{29,30}$

The area under the curve values obtained from receiver operating characteristic analysis for these 3 measures indicate that MUCCA, CCV, and TCV are not sufficiently sensitive or specific to be deemed clinically relevant diagnostic biomarkers in NMOSD. ${ }^{31}$ We believe that measuring MUCCA longitudinally could still indicate whether SCA or cord damage occurred. ${ }^{32}$

The similar performance of MUCCA compared with CCV and TCV could be because the cervical and upper thoracic cord levels are most commonly affected in patients with NMOSD. ${ }^{20,33}$ Also, all fibers of the thoracic and lumbar spinal cord eventually converge, passing through the cervical cord. Hence, the cervical portion will reflect the overall damage even from lower segments.

Our results showed no clinical associations with the spinal cord measures we investigated. The literature on the associations of clinical disability with spinal cord measures is mixed for NMOSD.

Two studies from the same group found very strong associations of MUCCA with clinical measures in MS and NMOSD. ${ }^{29,34}$ One other spinal cord study in NMOSD observed some nonlesion-related damage and demonstrated a significant correlation of MUCCA with the patient-determined disease steps, but did not report any statistical tests with EDSS. ${ }^{30}$

This discrepancy with our results could be due to ethnic, demographic, or cohort-selection differences. Another reason for this finding could be that the EDSS was developed for multiple sclerosis and might not be an ideal instrument reflecting NMOSD disease severity. ${ }^{35}$

We chose not to apply normalization to all spinal cord measures because no consensus has been reached on how to normalize SCA measures. ${ }^{36-39}$ Performing spinal cord measurements using different types of MR imaging sequences may require different normalization methods, which we did not investigate in this study.

Measurement of MUCCA, CCV, and TCV could be influenced by inflammation of the spinal cord, yielding larger values than the true cross-sectional areas. None of the patients in our cohort were imaged during an acute myelitis phase. Thus, no patient MRIs exhibited edema in any part of the myelon, which may have affected the spinal cord measurements.

Some limitations apply to our study. The study was performed in a relatively small sample of patients with AQP4-Ab+ only. We conducted this study in NMOSD because we expected a relatively strong difference in SCA measures compared with a healthy control group. We have limited this study cohort to patients with AQP4-Ab disease only to keep the patient cohort homogeneous, so damage mechanisms should be similar. Where the addition of patients with AQP4-Ab seronegativity would include patients with myelin oligodendrocyte glycoprotein antibody seropositivity, likely with different damage in the spinal cord. ${ }^{40,41}$ The main purpose of our study was to compare 3 possible measures of SCA and investigate whether the additional effort of full spinal cord analysis performs better than a simple MUCCA approach. Thus, we did not evaluate spinal cord lesion load or location in this study.

We are aware that sagittally oriented MR images are not ideal for detailed analysis of the spinal cord. Full spinal cord MR imaging is usually acquired in a sagittal orientation because larger portions of the spinal cord can be imaged compared with an axial orientation. Axially acquired spinal cord images would have been advantageous due to fewer partial volume effects. When one looks at the axial slices in Fig 1, it becomes clear that image resolution in the $2 \mathrm{D}$ axial plane is coarse. However, the active surface model used by the Jim software smooths out voxels and interpolates the slice spacing. It does not remove the spacing; rather, it fills in the gaps using an average from the signal coming from the contributing slices on either side. Thus, we believe the discrepancy between $3 \mathrm{D}$ and $2 \mathrm{D}$ images is minimized and the partial volume errors are decreased by this method. ${ }^{19}$ Moreover, the acquisition of axial images over the full spinal cord would have required excessively long scan times, and our results would not be comparable with the standard clinical setting. ${ }^{42}$

There has been sparse research into MR imaging biomarkers for patients with NMOSD. Those who have investigated deep gray matter and white matter tracts have shown little discernable difference between patients with AQP4-Ab + NMOSD and healthy controls. ${ }^{43,44}$ Thus, the decrease in spinal cord measures found in this study and others shows that SCA could be a suitable imaging biomarker in these patients.

Emerging quantitative MR imaging methods of white and gray matter segmentation in the spinal cord are now becoming clini- 
cally available. $^{8,20,39,45}$ Further studies will be required to show whether these new measures can better characterize SCA in the NMOSD context.

\section{CONCLUSIONS}

MUCCA shows similar performance for SCA evaluation in comparison with CCV and TCV. The hypothetical benefit of a more complete volumetric measurement could not be confirmed in our study. Therefore, in our opinion, MUCCA is an efficient and representative surrogate parameter for SCA analysis, at least in the context of patients with AQP4-Ab+ NMOSD.

MUCCA has 2 advantages: 1) being relatively easy to acquire, without the need for a dedicated spine sequence because 3D T1weighted cerebral images can be used for MUCCA measurement; and 2) the measurement is relatively simple and quick to perform. The pathogenesis of SCA in NMOSD is still poorly understood, and the disease is difficult to study due to its rarity. It is, thus, of great advantage to have an efficient and easy-to-standardize method in future longitudinal studies. Our study demonstrates that MUCCA measurements are a good representative marker of whole spinal cord SCA in patients with NMOSD.

\section{ACKNOWLEDGMENTS}

We thank all the patients and healthy control subjects for their participation. We would also like to acknowledge our MR imaging technicians, Cynthia Kraut and Susan Pikol, for their assistance in this research.

Disclosures: A.U. Brandt-UNRELATED: Board Membership: Motognosis; Consultancy: Biogen, Comments: Vision Study; Payment for Lectures Including Service on Speakers Bureaus: Teva Pharmaceutical Industries; Patents (Planned, Pending, or Issued), Comments: ocular coherence tomography analysis, perceptive computing, MS biomarkers; Stock/Stock Options: Motognosis. J. Bellmann-StroblUNRELATED: Payment for Lectures Including Service on Speakers Bureaus, Comments: payment for lectures on fatigue in 2016 and pregnancy and MS in 2017; Travel/Accommodations/Meeting Expenses Unrelated to Activities Listed, Comments: travel grants in 2015, 2016, and 2017. Klemens Ruprecht-RELATED: Grant: KKNMS (Competence Network Multiple Sclerosis) Bundesministerium für Bildung und Forschung, Comments: research grant; UNRELATED: Grants/Grants Pending: Novartis Pharmaceuticals, Merck Serono, Comments: research grants. Friedemann Paul—UNRELATED: Board Membership: Guthy Jackson Charitable Foundation-International Clinical Consortium. * ${ }^{*}$ Money paid to the institution.

\section{REFERENCES}

1. Filippi M, Rocca MA, Ciccarelli O, et al; MAGNIMS Study Group. MRI criteria for the diagnosis of multiple sclerosis: MAGNIMS consensus guidelines. Lancet Neurol 2016;15:292-303 CrossRef Medline

2. Pittock SJ, Lennon VA, Krecke K, et al. Brain abnormalities in neuromyelitis optica. Arch Neurol 2006;63:390-96 CrossRef Medline

3. Kim HJ, Paul F, Lana-Peixoto MA, et al; Guthy-Jackson Charitable Foundation NMO International Clinical Consortium \& Biorepository. MRI characteristics of neuromyelitis optica spectrum disorder: an international update. Neurology 2015;84:1165-73 CrossRef Medline

4. Asgari N, Flanagan EP, Fujihara K, et al. Disruption of the leptomeningeal blood barrier in neuromyelitis optica spectrum disorder. Neurol Neuroimmunol Neuroinflamm 2017;4:e343 CrossRef Medline

5. Schmidt F, Zimmermann H, Mikolajczak J, et al. Severe structural and functional visual system damage leads to profound loss of vision-related quality of life in patients with neuromyelitis optica spectrum disorders. Mult Scler Relat Disord 2017;11:45-50 CrossRef Medline

6. Oertel FC, Kuchling J, Zimmermann H, et al. Microstructural visual system changes in AQP4-antibody-seropositive NMOSD. Neurol Neuroimmunol Neuroinflamm 2017;4:e334 CrossRef Medline

7. Wingerchuk DM, Banwell B, Bennett JL, et al; International Panel for NMO Diagnosis. International consensus diagnostic criteria for neuromyelitis optica spectrum disorders. Neurology 2015;85: 177-89 CrossRef Medline

8. Pekcevik Y, Mitchell CH, Mealy MA, et al. Differentiating neuromyelitis optica from other causes of longitudinally extensive transverse myelitis on spinal magnetic resonance imaging. Mult Scler 2016;22:302-11 CrossRef Medline

9. Akaishi T, Nakashima I, Sato DK, et al. Neuromyelitis optica spectrum disorders. Neuroimaging Clin N Am 2017;27:251-65 CrossRef Medline

10. Wang Y, Wu A, Chen X, et al. Comparison of clinical characteristics between neuromyelitis optica spectrum disorders with and without spinal cord atrophy. BMC Neurol 2014;14:246 CrossRef Medline

11. Stevenson VL, Leary SM, Losseff NA, et al. Spinal cord atrophy and disability in MS: a longitudinal study. Neurology 1998;51:234-38 CrossRef Medline

12. Losseff NA, Webb SL, O'Riordan JI, et al. Spinal cord atrophy and disability in multiple sclerosis: a new reproducible and sensitive MRI method with potential to monitor disease progression. Brain 1996;119(Pt 3):701-08 CrossRef Medline

13. Daams M, Weiler F, Steenwijk MD, et al. Mean upper cervical cord area (MUCCA) measurement in long-standing multiple sclerosis: relation to brain findings and clinical disability. Mult Scler 2014;20: 1860-65 CrossRef Medline

14. Liu Z, Yaldizli Ö, Pardini M, et al. Cervical cord area measurement using volumetric brain magnetic resonance imaging in multiple sclerosis. Mult Scler Relat Disord 2015;4:52-57 CrossRef Medline

15. Liu Y, Lukas C, Steenwijk MD, et al. Multicenter validation of mean upper cervical cord area measurements from head 3D T1-weighted MR imaging in patients with multiple sclerosis. AJNR Am J Neuroradiol 2016;37:749-54 CrossRef Medline

16. Jarius S, Ruprecht K, Kleiter I, et al; in cooperation with the Neuromyelitis Optica Study Group (NEMOS). MOG-IgG in NMO and related disorders: a multicenter study of 50 patients, part 2-epidemiology, clinical presentation, radiological and laboratory features, treatment responses, and long-term outcome. J Neuroinflammation 2016;13:280 CrossRef Medline

17. Sato DK, Callegaro D, Lana-Peixoto MA, et al. Distinction between MOG antibody-positive and AQP4 antibody-positive NMO spectrum disorders. Neurology 2014;82:474-81 CrossRef Medline

18. Zekeridou A, Lennon VA. Aquaporin-4 autoimmunity. Neurol Neuroimmunol Neuroinflamm 2015;2:e110 CrossRef Medline

19. Horsfield MA, Sala S, Neema M, et al. Rapid semi-automatic segmentation of the spinal cord from magnetic resonance images: application in multiple sclerosis. Neuroimage 2010;50:446-55 CrossRef Medline

20. Kim G, Khalid F, Oommen VV, et al. T1- vs. T2-based MRI measures of spinal cord volume in healthy subjects and patients with multiple sclerosis. BMC Neurol 2015;15:124 CrossRef Medline

21. Castellano A, Papinutto N, Cadioli M, et al. Quantitative MRI of the spinal cord and brain in adrenomyeloneuropathy: in vivo assessment of structural changes. Brain 2016;139(Pt 6):1735-46 CrossRef Medline

22. Cawley N, Tur C, Prados F, et al. Spinal cord atrophy as a primary outcome measure in phase II trials of progressive multiple sclerosis. Mult Scler 2017 May 1. [Epub ahead of print] CrossRef Medline

23. Hajian-Tilaki K. Receiver operating characteristic (ROC) curve analysis for medical diagnostic test evaluation. Caspian J Intern Med 2013;4:627-35 Medline

24. R Core Team. R version 3.4.1. 2014. http://www.R-project.org/.

25. Wickham H. Tidyverse: Easily Install and Load the "Tidyverse." November 14, 2017. https://CRAN.R-project.org/package=tidyverse. Accessed April 27, 2018

26. Kassambara A. ggpubr: "ggplot2" Based Publication Ready Plots. 
November 14, 2017. https://cran.r-project.org/web/packages/ggpubr/ index.html. Accessed April 27, 2018

27. Robin X, Turck N, Hainard A, et al. pROC: an open-source package for $\mathrm{R}$ and $\mathrm{S}+$ to analyze and compare receiver operating characteristic curves. BMC Bioinformatics 2011;12:77 CrossRef Medline

28. Ontaneda D, Cohen JA, Amato MP. Clinical outcome measures for progressive MS trials. Mult Scler 2017;23:1627-35 CrossRef Medline

29. Liu Y, Wang J, Daams M, et al. Differential patterns of spinal cord and brain atrophy in NMO and MS. Neurology 2015;84:1465-72 CrossRef Medline

30. Ventura RE, Kister I, Chung S, et al. Cervical spinal cord atrophy in NMOSD without a history of myelitis or MRI-visible lesions. Neurol Neuroimmunol Neuroinflamm 2016;3:e224 CrossRef Medline

31. Skates SJ. A statistical challenge: developing tests for biomarker utility specific to the intended use. J Natl Cancer Inst 2014;106: dju076 CrossRef Medline

32. Chen W, Samuelson FW, Gallas BD, et al. On the assessment of the added value of new predictive biomarkers. BMC Med Res Methodol 2013;13:98 CrossRef Medline

33. Wang $Y$, Wang $Y$, Tan $S$, et al. Spinal cord atrophy in neuromyelitis optica spectrum disorders. Mult Scler Relat Disord 2016;8:9-10 CrossRef Medline

34. Liu Y, Duan Y, Huang J, et al. Different patterns of longitudinal brain and spinal cord changes and their associations with disability progression in NMO and MS. Eur Radiol 2018;28:96-103 CrossRef Medline

35. Cree BA, Bennett JL, Sheehan M, et al. Placebo-controlled study in neuromyelitis optica: ethical and design considerations. Mult Scler 2016;22:862-72 CrossRef Medline

36. Healy BC, Arora A, Hayden DL, et al. Approaches to normalization of spinal cord volume: application to multiple sclerosis. J Neuroimaging 2012;22:e12-19 CrossRef Medline
37. Oh J, Seigo M, Saidha S, et al. Spinal cord normalization in multiple sclerosis. J Neuroimaging 2014;24:577-84 CrossRef Medline

38. Kearney H, Yiannakas MC, Abdel-Aziz K, et al. Improved MRI quantification of spinal cord atrophy in multiple sclerosis. J Magn Reson Imaging 2014;39:617-23 CrossRef Medline

39. Papinutto N, Schlaeger R, Panara V, et al. Age, gender and normalization covariates for spinal cord gray matter and total cross-sectional areas at cervical and thoracic levels: a 2D phase sensitive inversion recovery imaging study. PLoS One 2015;10:e0118576 CrossRef Medline

40. Zamvil SS, Slavin AJ. Does MOG Ig-positive AQP4-seronegative opticospinal inflammatory disease justify a diagnosis of NMO spectrum disorder? Neurol Neuroimmunol Neuroinflamm 2015;2:e62 CrossRef Medline

41. Jarius S, Ruprecht K, Kleiter I, et al; in cooperation with the Neuromyelitis Optica Study Group (NEMOS). MOG-IgG in NMO and related disorders: a multicenter study of $\mathbf{5 0}$ patients, Part 1: frequency, syndrome specificity, influence of disease activity, long-term course, association with AQP4-IgG, and origin. J Neuroinflammation 2016;13:279 CrossRef Medline

42. Ozturk A, Aygun N, Smith SA, et al. Axial 3D gradient-echo imaging for improved multiple sclerosis lesion detection in the cervical spinal cord at 3T. Neuroradiology 2013;55:431-39 CrossRef Medline

43. Finke C, Heine J, Pache F, et al. Normal volumes and microstructural integrity of deep gray matter structures in AQP4+ NMOSD. Neurol Neuroimmunol Neuroinflamm 2016;3:e229 CrossRef Medline

44. Pache F, Zimmermann $\mathrm{H}$, Finke $\mathrm{C}$, et al. Brain parenchymal damage in neuromyelitis optica spectrum disorder: a multimodal MRI study. Eur Radiol 2016;26:4413-22 CrossRef Medline

45. Dupont SM, De Leener B, Taso M, et al. Fully-integrated framework for the segmentation and registration of the spinal cord white and gray matter. Neuroimage 2017;150:358-72 CrossRef Medline 